\title{
Avaliação de Uma Escala de Risco em Pacientes Submetidos à Cirurgia de Revascularização do Miocárdio. Análise de 400 Casos*
}

\author{
Assessment of a Risk Scale in Patients Submitted to \\ Coronary Artery Bypass Surgery. Analysis of 400 Cases
}

Hélcio Giffhorn ${ }^{1}$

\section{RESUMO}

JUSTIFICATIVA E OBJETIVOS: O objetivo deste estudo foi o de avaliar uma escala de risco baseada em um protocolo desenvolvido na The Cleveland Clinic Foundation para procedimento cirúrgico de revascularização do miocárdio (RM), utilizando os parâmetros de pré e peri-operatório e o quadro fisiológico do paciente na admissão à unidade de terapia intensiva (UTI).

MÉTODO: No período de maio de 1999 a janeiro de 2002, parte dos pacientes submetidos a procedimento cirúrgico de RM no Serviço de Cirurgia Cardiovascular do Hospital Universitário Cajuru da Pontifícia Universidade Católica do Paraná, foram incluídos no protocolo de avaliação do grau de risco operatório, de modo prospectivo e consecutivo. Na avaliação pré-operatória um parâmetro $\leq$ a 6 foi associado a casos menos graves e parâmetros $>$ de 7 corresponderam a casos mais graves. Para a avaliação na admissão à UTI, esta

1. Cirurgião Cardiovascular/Medicina Intensiva do Hospital Instituição. Hospital Universitário Cajuru da Pontifícia Universidade Católica do Paraná, Curitiba, PR.

*Recebido do Serviço de Cirurgia Cardiovascular da Universidade Federal do Paraná, Curitiba, PR

- Estudo utilizado para dissertação de mestrado na universidade Federal do Paraná, tendo sido aprovada no dia 09/09/2002 com conceito A.

Apresentado em 16 de outubro de 2007

Aceito para publicação em 20 de fevereiro de 2008

Endereço para correspondência:

Dr.Hélcio Giffhorn

Rua Gastão Câmara, 694/ 1206 - Bigorrilho

80730-300 Curitiba, PR

Fone: (41) 3336-6251

E-mail: hgiffhorn@uol.com.br

(C)Associação de Medicina Intensiva Brasileira, 2008 classificação foi associada a valores de parâmetros $\leq$ a $14 \mathrm{e}>$ de 14. A morbidade foi classificada em complicações maiores e menores. Foram incluídos todos os pacientes em que foi possível obter o registro de todas as variáveis do protocolo.

RESULTADOS: Foram avaliados 400 pacientes que fizeram cirurgia com e sem o uso da circulação extracorpórea (CEC). Os fatores de risco pré-operatórios mais encontrados foram a idade entre 65 e 74 anos em 111 casos (27,75\%), peso abaixo de $65 \mathrm{~kg}$ em 106 (26,5\%), diabete melito em tratamento em 89 (22,25\%). O tempo de CEC foi acima de 160 minutos em 13 casos (6,95\%) e o balão intra-aórtico utilizado em 11 (2,75\%). No quadro fisiológico de admissão à UTI, a diferença alvéolo-arterial igual ou acima de $250 \mathrm{mmHg}$ foi o parâmetro mais encontrado em 334 pacientes (83,5\%), o bicarbonato de sódio arterial abaixo de $21 \mathrm{mmol} / \mathrm{L}$ foi identificado em 265 pacientes $(66,25 \%)$, e o desempenho cardiovascular marginal ou inadequado foi observado em 263 pacientes (65,75\%). Complicações maiores ocorreram em 124 pacientes (31\%) e a síndrome de baixo débito foi mais freqüente em $64(51,61 \%)$. Foram registrados 22 óbitos (5,5\%), sendo os de etiologia cardiovascular os mais freqüentes (40,9\%). Em 370 pacientes $(92,5 \%)$ a avaliação pré-operatória teve como resultado um parâmetro $\leq 6$; e, no momento da admissão à UTI, o parâmetro foi $\leq$ a 14 em 345 casos $(86,25 \%)$. Os pacientes com parâmetros maiores, tanto no pré-operatório quanto no momento da admissão à UTI, apresentaram mais complicações e maior ocorrência de óbito.

CONCLUSÕES: Os parâmetros registrados no momento pré-operatório e no momento da admissão à UTI apresentaram correlação com a probabilidade de óbito e de complicações do paciente e que o parâmetro na admissão à UTI, associado ao de pré e ao perioperatório é mais informativo tanto para o prognóstico 
de óbito quanto de complicações. Os modelos ajustados expressaram adequadamente as probabilidades estimadas de óbito e de complicações, em função dos parâmetros no pré-operatório, peri-operatório e na admissão à UTI.

Unitermos: cirurgia, cirurgia de revascularização do miocárdio, revascularização.

\section{SUMMARY}

BACKGROUND AND OBJECTIVES: The objective of this paper was to assess a risk scale based on a protocol developed at The Cleveland Clinic Foundation for the coronary artery bypass surgery using preoperative and perioperative parameters and the patient's physiological profile at the admission in the intensive care unit (ICU).

METHODS: In the period between May,1999 and January,2002 part of the patients who underwent coronary artery bypass surgery in the cardiac service of the Hospital Universitário Cajuru of Pontifícia Universidade Católica do Paraná were included in the assessment of the operative risk scale, prospectively and consecutive. During the postoperative assessment a parameter $\leq 6$ was associated to less severe cases and parameters above 7 corresponding to more severe cases. For the evaluation of ICU admission, this classification was associated with parameter values $\leq$ and $>14$. Morbidity was classified in major complications as well as less important complications. There were including all patients that it was possible to include all variables of the protocol.

RESULTS: Four hundred patients who underwent surgery, with cardiopulmonary bypass or off-pump coronary bypass, were assessed. There was 22 deaths (5.5\%) and 124 patients with major complications (31\%). The most common preoperative risk factors were the age between 65 and 74 in 111 cases (27.75\%), weight below $65 \mathrm{~kg}$ in $106(26.5 \%)$. Diabetes mellitus being treated in 89 cases $(22.5 \%)$. The cardiopulmonary bypass time was above 160 minutes in 13 cases (6.95\%) and intraaortic balloon pump was used in 11 (2.75\%). During the analysis of the ICU admission physiology, the alveolar arterial gradient equal or above $250 \mathrm{mmHg}$ was the parameter found in 334 patients (83.5\%), arterial sodium bicarbonate at ICU below $21 \mathrm{mmol} / \mathrm{L}$ was identified in 265 patients $(66.25 \%)$, and the marginal or inadequate cardiovascular performance was observed in 263 patients $(65.75 \%)$. The major complications occurred in 124 patients $(31 \%)$ and the low output syndrome was more frequently seen in $64(51.61 \%)$ of the cases. Twenty-two deaths were recorded (5.5\%) and the ones with cardiovascular etiology were the most frequent $(40.9 \%)$. In 370 patients $(92.5 \%)$ the preoperative assessment showed as a result a parameter equal or below 6 and the ICU admission, the parameter was equal or below 14 in 345 cases $(86.25 \%)$. Patients with parameters above that, at the preoperative and at the ICU admission, have shown more complications and more deaths.

CONCLUSIONS: It was possible to conclude that the parameters recorded at the preoperative and at the ICU admission were correlated with the probability of death and complications and the ICU admission parameter, associated with the preoperative parameters and intraoperative factors bring more information for the prognostic of death and complications. The adjusted models adequately express the death and complications probabilities estimate, due to the preoperative, intraoperative and ICU admission parameters.

Key Words: coronary artery bypass surgery, revascularization, surgery.

\section{INTRODUÇÃO}

A partir de 1967, a cirurgia de revascularização do miocárdio tem apresentado grande desenvolvimento, principalmente após os estudos de Favaloro em Cleveland, Ohio ${ }^{1,2}$, quando utilizou o emprego da veia safena em posição reversa entre a aorta ascendente e a artéria coronária direita.

Inicialmente com o auxílio da circulação extracorpórea (CEC) e após sem o seu emprego ${ }^{3,4}$, a revascularização do miocárdio (RM) tornou-se a intervenção cirúrgica mais freqüente entre as cardiopatias. A evolução tecnológica e médica no atendimento pré, peri e pós-operatório diminuíram a morbimortalidade e melhoraram a qualidade de vida dos pacientes, sendo, entretanto, acompanhada por uma elevação nos custos com o tratamento da saúde ${ }^{5}$. Muitos estudos foram desenvolvidos para a melhor orientação de condutas para a $\mathrm{RM}^{6,7}$, apesar disso, não haviam critérios padronizados para a avaliação dos resultados operatórios ${ }^{8,9,10}$.

Desde os anos 1980, buscaram-se índices para a compilação e posterior análise de resultados dos pacientes submetidos à procedimento cardíaco ${ }^{11}$.

Entretanto, estes programas de avaliação de risco operatório não levavam em conta dados de peri-operatório e da admissão do paciente na Unidade de Terapia Intensiva (UTI).

Em 1997, na The Cleveland Clinic Foudation, iniciou-se 
um protocolo com uma avaliação para o risco de morbimortalidade levando-se em consideração os parâmetros de pré-operatório, peri-operatório e do quadro fisiológico do paciente admitido na UTI'12. Este protocolo trouxe um significativo avanço na avaliação de risco para os pacientes submetidos à RM, porque todos os protocolos anteriores somente incluíam parâmetros de avaliação pré-operatória.

O objetivo deste estudo foi o de adequar uma escala de risco em pacientes submetidos a RM baseado no protocolo desenvolvido na The Cleveland Clinic Foundation para avaliar a aplicabilidade deste modelo para risco de complicações e óbito; avaliar a precisão dos parâmetros de pré-operatório associados ou não aos de peri-operatório e da admissão à UTI para quantificação dos riscos nestes pacientes.

\section{MÉTODO}

Após aprovação do Comitê de Ética e Pesquisa dos Hospitais da Aliança Saúde da Pontifícia Universidade Católica da Paraná, no período de maio de 1999 a janeiro de 2002, parte dos pacientes submetidos à RM no serviço de Cirurgia Cardíaca do Hospital Universitário Cajuru da Pontifícia Universidade Católica do Paraná (HUC) foram incluídos no protocolo de avaliação do grau de risco operatório (Tabela 1), de modo prospectivo e consecutivo. Com o registro das variáveis do protocolo, foi possível a determinação de parâmetros numa escala de 0 a 31 para o pré-operatório e de 0 a 45 na admissão à UTI. Por meio do parâmetro avaliado no momento pré-operatório, cada paciente foi classificado como sendo de baixo risco operatório (parâme-

Tabela 1 - Protocolo de Escala de Risco para Pacientes Submetidos à Cirurgia de Revascularização do Miocárdio

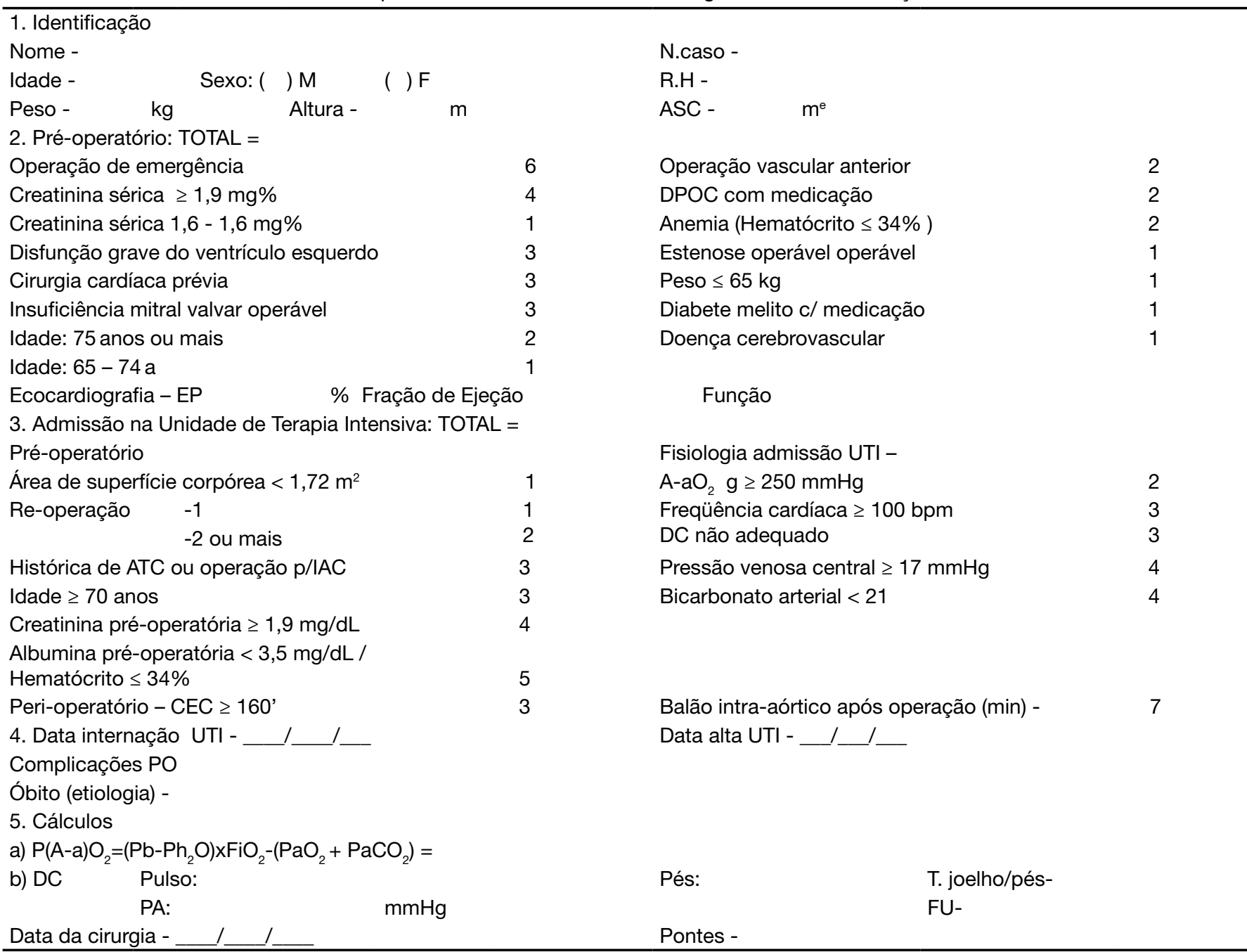

$\mathrm{g}$ = gradiente; $\mathrm{DPOC}=$ doença pulmonar obstrutiva crônica; EP = encurtamento percentual; ATC = angioplastia transluminal coronária; IAC = insuficiência arterial crônica $(\mathrm{mg} / \mathrm{dL}) ; \mathrm{A}-\mathrm{aO}^{2}=$ diferença alvéolo-arterial de $\mathrm{O}_{2} ; \mathrm{DC}=$ desempenho cardiovascular não adequado (marginal ou inadequado); $\mathrm{CEC}=$ circulação extracorpórea; $\mathrm{PO}=$ pós-operatório; $\mathrm{T}=$ temperatura $\left({ }^{\circ} \mathrm{C}\right) ; \mathrm{PA}=$ pressão arterial; $\mathrm{FU}=$ fluxo urinário $(\mathrm{mL} / \mathrm{kg} / \mathrm{h})$ 
tro $\leq 6)$ ou de alto risco operatório (parâmetro $>6$ ). $\mathrm{Na}$ avaliação no momento da admissão à UTI esta mesma classificação correspondeu a parâmetro de $\leq 14$ e > 14, respectivamente.

Foram incluídos neste estudo 400 pacientes, sendo possível obter o registro de todas as variáveis do protocolo no momento pré-operatório e na primeira hora de admissão à UTI.

\section{Definição de Variáveis do Protocolo}

Área de superfície corporal - utilizou-se o nomograma de DuBois para a determinação da área de superfície corporal em adultos ${ }^{13}$.

Creatinina sérica elevada - entre 1,6 e 1,8mg\% de menor gravidade ; igual ou acima a $1,9 \mathrm{mg} \%$ de maior gravidade.

Cirurgia de emergência - caracterizada por necessidade de operação imediata após insucesso de angioplastia coronária percutânea ou quando a operação era necessária até $24 \mathrm{~h}$ após a realização de estudo cineangiocoronariográfico diagnóstico, lesão de coronária esquerda ou angina instável intratável ao manuseio clínico.

Disfunção do ventrículo esquerdo - foram utilizados os dados por meio da ecocardiografia e do ventriculograma esquerdo.

Ecocardiografia - avaliação da fração de ejeção, do encurtamento percentual e função cardíaca, valores estes obtidos no serviço de ecocardiografia do HUC e calculados por métodos descritos por vários auto$\operatorname{res}^{14-16}$ (Anexos 1,2).

Cateterismo cardíaco - avaliação por meio do ventriculograma esquerdo e sua contratilidade segmentar ${ }^{17-18}$ :

- Acinesia - ausência total de movimento do segmento;

- Hipocinesia - diminuição do movimento esperado;

- Discinesia - expansão sistólica paradoxal do segmento (aneurisma ventricular);

- Normal - sem alterações de contratilidade segmentar. Insuficiência mitral operável - pacientes com insuficiência mitral com indicação de cirurgia: presença de refluxo moderado ou intenso de sangue ou contraste, que se traduz com opacificação completa tanto do átrio, como do ventrículo esquerdo ${ }^{19}$.

Idade - paciente com idade entre 65 e 74 anos ou com 75 anos ou mais.

Anemia - considerada presente quando o hematócrito estava menor ou acima a $34 \%$.

Estenose aórtica operável - pacientes com estenose aórtica moderada ou grave, sintomáticos ou com área valvar de $0,75 \mathrm{~cm}^{2}$ ou menos ${ }^{20}$.
Peso - paciente com peso menor ou igual a $65 \mathrm{~kg}$.

Area de superfície corporal - maior risco quando a área é menor a $1,72 \mathrm{~m}^{2}$.

Re-operação - paciente com uma indicação de reoperação ou que foram submetidos a duas ou mais.

História anterior de angioplastia coronariana ou com história de angioplastia ou operação para insuficiência arterial periférica .

Albumina sérica pré-operatória abaixo de $3,5 \mathrm{mg} / \mathrm{dL}$ ou hematócrito menor ou igual a $34 \%$ - considerouse para avaliação deste trabalho somente o valor do hematócrito.

CEC igual ou acima a 160' - tempo de circulação extracorpórea igual ou acima de 160 minutos. BIAo após operação - considerou-se o uso de balão intra-aórtico após a circulação extracorpórea ou quando necessário na revascularização do miocárdio sem circulação extracorpórea; sua utilização era em caso de choque cardiogênico, síndrome de baixo débito cardíaco após cardiotomia e diminuição importante da função ventricular esquerda ${ }^{21}$.

Doenças associadas - em pacientes que fazem uso de medicações: diabete melito insulino dependente ou não, doença pulmonar obstrutiva crônica e para os pacientes com antecedentes de tratamento de doença vascular periférica ou cérebro-vascular.

Diferença alvéolo-arterial de oxigênio $\left(P(A-a) O_{2}\right)$ - empregada como um índice de eficiência das trocas gasosas $^{22}$. Foi considerado anormal se igual ou acima de $250 \mathrm{mmHg}$ (Anexo 3).

Desempenho cardiovascular - avaliado de acordo com critérios clínicos e classificado em adequado, marginal ou inadequado (Anexos 4,5,6,7).

\section{Morbidade}

Foi definida como a presença de complicações maiores ou menores ocorridas no período do pósoperatório:

Complicações maiores - complicações que ocorreram e que impuseram risco ao paciente no período de pósoperatório imediato classificadas em:

- Cardiovascular - síndrome de baixo débito cardíaco, edema agudo de pulmão, disritmias ventriculares (taquicardia ventricular, fibrilação ventricular), infarto agudo do miocárdio no pós-operatório e assistolia cardíaca;

- Respiratória - insuficiência respiratória aguda, hemorragia pulmonar, tromboembolismo pulmonar, síndrome da angústia respiratória do adulto;

- Renal - insuficiência renal aguda; 
- Gastrintestinal - não ocorreu;

- Sistema nervoso central - acidente vascular encefálico;

- Outras complicações maiores - disfunção de múltiplos órgãos; hemorragia pós-operatória (necessitando de re-operação); mediastinite; síndrome inflamatória pós-circulação extracorpórea; sepse; hipercalemia.

Complicações menores - complicações ocorridas , mas que não implicaram em risco de vida ao paciente no pós-operatório imediato e que não foram consideradas como fator de morbidade nesta avaliação de risco cirúrgico, sendo classificadas em:

- Cardiovasculares - disritmias (taquicardia supraventricular, fibrilação atrial, bloqueio atrioventricular temporário, extrassístoles ventriculares), hipertensão arterial sistêmica, pericardite pós-pericardiectomia, insuficiência cardíaca esquerda;

- Respiratória - fístula broncopleural, broncopneumonia, pneumotórax, derrame pleural;

- Renal - infecção do trato urinário;

- Gastrointestinal - hemorragia digestiva alta;

- Sistema nervoso central - crises convulsivas;

- Outras complicações menores - deiscência esternal; síndrome de abstinência; depleção do espaço extracelular.

\section{Pré-Operatório}

Solicitava-se rotina pré-operatória após a internação do paciente (medicações e exames complementares). Realizava-se a avaliação do tipo de enxerto a ser utilizado após o estudo hemodinâmico e o biotipo do paciente.

\section{Anestesia}

Após a monitorização cardíaca e o preparo do paciente, a indução anestésica foi feita por via venosa, utilizando-se midazolam, citrato de fentanil, brometo de pancurônio e isoflurano como agente inalatório. Para a manutenção anestésica utilizou-se metade das doses dos fármacos empregados.

\section{Cirurgia}

Toracotomia mediana por planos e retirada simultânea dos enxertos arteriais e venosos. Foi realizada heparinização sistêmica do paciente. Na RM sem CEC foi utilizado o ponto de Lima para tração do pericárdio parietal posterior a fim de facilitar a exposição cardíaca. Quando a CEC era empregada, o pinçamento aórtico podia ou não ser realizado, após a indução da fibri- lação ventricular com hipotermia sistêmica e fibrilador cardíaco.

Realizavam-se as anastomoses proximais e distais, administração de sulfato de protamina e procedia-se a hemostasia torácica e das incisões. O paciente era então encaminhado à UTI.

\section{UTI}

Após a admissão do paciente na UTI, realizava-se a solicitação dos exames complementares, orientação para o pós-operatório e o preenchimento do protocolo de avaliação da escala de risco, na primeira hora da chegada na UTI (Tabela 1).

\section{Análise Estatística}

Foi utilizado para a análise estatística o modelo de regressão logística (teste de Wald), teste de razão das verossimelhanças, teste de diferença de proporções e estatística descritiva. $O$ valor de $p<0,05$ foi considerado estatisticamente significativo.

Para a investigação da influência do parâmetro de préoperatório, peri-operatório e dos parâmetros na admissão na UTI sobre a probabilidade de óbito e de complicações, ajustou-se o modelo de Regressão Logística e aplicou-se o teste de Wald.

Para verificar se o parâmetro pré-operatório identificou significativamente o prognóstico de óbito do paciente, considerou-se o teste da Razão de verossimelhanças para comparar o modelo de Regressão Logística ajustado, considerando-se somente o parâmetro préoperatório. A fim de investigar se o parâmetro pré-operatório influencia ou não a necessidade de CEC durante a operação, foi utilizado o modelo de Regressão Logística.

Para as demais variáveis observadas e registradas dos pacientes, foram apresentadas estatísticas descritivas. Em toda a análise, o valor de $p<0,05$ foi considerado estatisticamente significativo.

\section{RESULTADOS}

Os dados pré-operatórios mais freqüentes foram a idade entre 65 e 74 anos em 111 casos $(27,75 \%)$, peso $\leq 65 \mathrm{~kg}$ em 106 (26,5\%), diabete melito com uso de medicação em 89 (22,25\%) e anemia com hematócrito $\leq 34 \%$ (Tabela 2). A ecocardiografia foi realizada no pré-operatório em 364 pacientes (91\%). A escala de risco pré-operatória com pacientes de parâmetro $\leq 6$ ocorreu em 370 casos $(92,5 \%)$ e $>6$ em 30 (7,5\%) (Tabela 4). 
Tabela 2 - Escala de Fatores de Risco na Fase Pré-Operatória.

\begin{tabular}{|c|c|c|c|}
\hline \multicolumn{2}{|l|}{ Fatores de Risco } & $\begin{array}{l}\mathrm{N}^{\circ} \text { de } \\
\text { Casos }\end{array}$ & $\%$ \\
\hline \multicolumn{2}{|c|}{ Cirurgia de emergência } & 26 & 6,5 \\
\hline \multirow[t]{2}{*}{ Creatinina sérica } & $\geq 1,9 \mathrm{mg} \%$ & 17 & 4,25 \\
\hline & $1,6-1,9 \mathrm{mg} \%$ & 20 & 5,0 \\
\hline \multicolumn{2}{|c|}{ Disfunção grave do ventrículo esquerdo } & 35 & 8,75 \\
\hline \multicolumn{2}{|c|}{ Operação cardíaca prévia } & 22 & 5,5 \\
\hline \multicolumn{2}{|c|}{ Insuficiência mitral valvar operável } & 06 & 1,5 \\
\hline \multirow[t]{2}{*}{ Idade } & 75 anos ou mais & 42 & 10,5 \\
\hline & 65 a 74 anos & 111 & 27,75 \\
\hline \multicolumn{2}{|c|}{ Operação vascular anterior } & 13 & 3,25 \\
\hline \multicolumn{2}{|c|}{ DPOC com medicação } & 08 & 2,0 \\
\hline \multicolumn{2}{|c|}{ Anemia (hematócrito $\leq 34 \%$ ) } & 47 & 11,75 \\
\hline \multicolumn{2}{|c|}{ Estenose aórtica operável } & 05 & 1,25 \\
\hline \multicolumn{2}{|l|}{ Peso $\leq 65 \mathrm{~kg}$} & 106 & 26,5 \\
\hline \multicolumn{2}{|c|}{ Diabete melito com medicação } & 89 & 22,25 \\
\hline \multicolumn{2}{|c|}{ Doença cerebrovascular } & 35 & 8,78 \\
\hline
\end{tabular}

Os dados de admissão na UTI mais freqüentes no préoperatório foram área de superfície corporal abaixo de $1,72 \mathrm{~m}^{2}$ que ocorreu em 127 pacientes (31,75\%), história de angioplastia ou cirurgia para insuficiência arterial crônica e hematócrito menor ou abaixo de $34 \%$, ambos em 47 casos $(11,75 \%)$. Na fase peri-operatória o tempo de CEC foi igual ou maior a $160 \mathrm{~min}$ em 13 (6,95\%). $\mathrm{Na}$ fisiologia de admissão na UTI, a diferença alvéoloarterial de oxigênio encontrava-se igual ou acima a 250 mmHg em 334 pacientes (83,5\%), o bicarbonato arterial abaixo de $21 \mathrm{mmol} / \mathrm{L}$ em 265 (66,25\%) e o desempenho cardiovascular não adequado em 263 (65,75\%) (Tabela 3). A escala de risco na admissão $\leq 14$ em 345 pacientes (86,25\%) e acima de 14 em 55 (13,75\%) (Tabela 4).

Tabela 3 - Escala de Fatores de Risco na Admissão à UTI.

\begin{tabular}{|c|c|c|}
\hline Fatores de Risco & $\begin{array}{l}\text { Número } \\
\text { de Casos }\end{array}$ & $\%$ \\
\hline \multicolumn{3}{|l|}{ Pré-operatório } \\
\hline Área de superfície corpórea $<1,72 \mathrm{~m}^{2}$ & 127 & 31,75 \\
\hline \multirow[t]{2}{*}{ Re-operação } & 22 & 5,5 \\
\hline & 00 & 0,0 \\
\hline História de ATC ou operação IAC & 47 & 11,75 \\
\hline Idade $\geq 70$ anos & 96 & 24 \\
\hline Creatinina $\geq 1,9 \mathrm{mg} / \mathrm{dL}$ & 17 & 4,25 \\
\hline Anemia com hematócrito $\leq 34 \%$ & 47 & 11,75 \\
\hline \multicolumn{3}{|l|}{ Peri-operatório } \\
\hline Tempo de CEC $\geq 160$ minutos & 13 & 6,75 \\
\hline BIA após operação & 11 & 2,75 \\
\hline \multicolumn{3}{|l|}{ Filosofia à admissão na UTI } \\
\hline $\mathrm{A}-\mathrm{aO}_{2}(\mathrm{~g} \geq 250 \mathrm{mmHg})$ & 334 & 83,5 \\
\hline Freqüência cardíaca $\geq 100 \mathrm{bpm}$ & 162 & 40,5 \\
\hline Débito cardíaco não adequado & 263 & 65,75 \\
\hline Pressão venosa central $\geq 17 \mathrm{mmHg}$ & 29 & 7,25 \\
\hline Bicarbonato arterial $<21$ & 265 & 66,25 \\
\hline
\end{tabular}

ATC = angioplastia transluminal coronária; IAC = insuficiência arterial crônica; $\mathrm{CEC}=$ circulação extracorpórea; $\mathrm{BIAO}=$ balão intra-aórtico; $\mathrm{A}-\mathrm{aO}_{2}=$ diferença alvéolo-arterial de oxigênio
Tabela 4 - Complicações Maiores e os Parâmetros de Avaliação de Risco.

\begin{tabular}{|c|c|}
\hline Parâmetro Pré-Operatório & Parâmetro à Admissão na UTI \\
\hline Pré-operatório $\leq 6 \mathrm{n}=370$ & Admissão à UTI $\leq 14 \mathrm{n}=316$ \\
\hline \multirow{3}{*}{$\begin{array}{l}111 \text { pacientes com complica- } \\
\text { ções maiores } \\
\% \text { complicações maiores: } \\
30,00 \%\end{array}$} & $\begin{array}{l}80 \text { pacientes com complica- } \\
\text { ções maiores }\end{array}$ \\
\hline & $\begin{array}{l}\% \text { complicações maiores: } \\
25,32 \%\end{array}$ \\
\hline & $\begin{array}{l}\text { Admissão à UTI > } 14 \mathrm{n}=54 \\
31 \text { pacientes com complica- } \\
\text { ções maiores } \\
\text { \% complicações: } 57,41 \%\end{array}$ \\
\hline \multirow{6}{*}{$\begin{array}{l}\text { Pré-operatório }>6 n=30 \\
13 \text { pacientes com complica- } \\
\text { ções maiores } \\
\% \text { complicações maiores: } \\
43,33 \%\end{array}$} & Admissão à UTI $\leq 14 \mathrm{n}=13$ \\
\hline & $\begin{array}{l}5 \text { pacientes com complicações } \\
\text { maiores }\end{array}$ \\
\hline & $\begin{array}{l}\% \text { complicações maiores: } \\
38,46 \%\end{array}$ \\
\hline & Admissão à UTI > 14 n = 17 \\
\hline & $\begin{array}{l}8 \text { pacientes com complicações } \\
\text { maiores }\end{array}$ \\
\hline & $\begin{array}{l}\% \text { complicações maiores: } \\
47,06 \%\end{array}$ \\
\hline
\end{tabular}

Para a análise das complicações, foram consideradas maiores aquelas que impuseram risco ao paciente no pós-operatório imediato. Ocorreram em 124 pacientes, representando $31 \%$ do total. Dentre essas, as mais freqüentes foram a síndrome de baixo débito cardíaco em 64 casos $(51,61 \%)$, insuficiência respiratória em 14 casos $(11,29 \%)$, insuficiência renal aguda em 10 casos $(8,06 \%)$, acidente vascular isquêmico em 18 (14,51\%) e re-operações para revisão de hemostasia em 12 casos $(9,67 \%)$. A presença de complicações maiores em relação aos dados de avaliação de risco encontra-se na tabela 4.

Ao investigar a influência do resultado do parâmetro de avaliação de risco pré-operatório, não se rejeitou a hipótese nula de que a probabilidade de complicação com o parâmetro pré $\leq 6$ é igual à probabilidade de complicação com o parâmetro pré $>6(p=0,1339)$. Entretanto, ao observar-se a hipótese nula de que a probabilidade de complicação com o parâmetro na admissão $\leq 14$ é igual à probabilidade de complicação com o parâmetro de admissão $>14$, o resultado do teste estatístico indicou a rejeição da hipótese nula no nível significativo de $5 \%(p<0,0001)$.

A fim de analisar o resultado conjunto dos parâmetros pré-operatórios, peri-operatórios e de admissão à UTI, observou-se a hipótese nula de que a inclusão do parâmetro de peri-operatório e de admissão não tem influência na probabilidade de complicação na presença do parâmetro pré-operatório. Esta hipótese foi rejeitada no nível de significância de $5 \%$ ( $p=$ 
0,000010). Desta forma, a inclusão dos parâmetros de peri-operatório e de admissão à UTI melhoraram significativamente o prognóstico sobre a probabilidade de complicação do paciente.

Ao ajustar o modelo com a utilização dos parâmetros pré, peri-operatórios e de admissão à UTI, foi possível estimar as probabilidades de complicações pós-operatórias do paciente, de acordo com os registros de parâmetros de risco nos dois momentos da avaliação.

$\mathrm{Na}$ tabela 6 estão apresentadas as probabilidades estimadas pelo modelo e as proporções observadas no estudo. Uma análise desses resultados em conjunto com os valores de $p$ correspondentes aos testes estatísticos realizados, permitiu concluir que o modelo estima adequadamente as probabilidades de complicações maiores do paciente quando conhecidos os valores de seus parâmetros de pré-operatório, peri-operatórios e na admissão à UTI. Além disso, observa-se que, quando o paciente apresenta parâmetros na admissão à UTI mais favoráveis, ou seja, $\leq 14$, qualquer que tenha sido o seu parâmetro no pré-operatório, a sua probabilidade estimada de complicações é bem menor do que quando apresenta parâmetros de maior risco (> 14) na admissão à UTI. Dos 400 casos estudados, ocorreram 22 óbitos $(5,5 \%)$. Desses, as causas cardiovasculares corresponderam a nove casos (síndrome de baixo débito cardíaco com sete, disritmias com dois (fibrilação ventricular com um caso e assistolia com um caso) (40,9\%); etiologia respiratória em três (insuficiência respiratória aguda) (13,63\%); insuficiência renal aguda em um caso (4,54\%), acidente vascular encefálico em um caso $(4,54 \%)$ e outras complicações em oito casos, ou seja: disfunção de múltiplos órgãos em três, hemorragia pós-operatória em dois, sepse em dois, e hipercalemia em um) (36,36\%). A ocorrência de óbitos em relação aos dados de avaliação de risco encontra-se na tabela 5.

Tabela 5 - Óbitos.

\begin{tabular}{ll}
\hline Parâmetros Pré-Operatórios & Parâmetros à Admissão na UTI \\
\hline Pré-operatório $\leq 6 \mathrm{n}=370$ & Admissão na UTI $\leq 14 \mathrm{n}=316$ \\
Óbitos - $18(4,86 \%)$ & Óbitos - $12(3,80 \%)$ \\
& Admissão na UTI > 14 $\mathrm{n}=54$ \\
& Óbitos - $6(11,11 \%)$ \\
Pré-operatório $>6 \mathrm{n}=30$ & Admissão na UTI $\leq 14 \mathrm{n}=13$ \\
& Óbito - $0(0,00 \%)$ \\
& Admissão na UTI > 14 $\mathrm{n}=17$ \\
& Óbitos - 4 (23,53\%) \\
\hline
\end{tabular}

Tabela 6 - Probabilidade de Complicação Estimada e Proporção de Complicação Observada.

\begin{tabular}{lcc}
\hline Parâmetros & $\begin{array}{c}\text { Probabilidade } \\
\text { de Complicação } \\
\text { Estimada }\end{array}$ & $\begin{array}{c}\text { Proporção de } \\
\text { Complicação } \\
\text { Observada }\end{array}$ \\
\hline Pré $\leq 6$ Admissão $\leq 14$ & 0,2579 & 0,2532 \\
Pré $\leq 6$ Admissão $>14$ & 0,5461 & 0,5741 \\
Pré $>6$ Admissão $\leq 14$ & 0,2684 & 0,3846 \\
Pré $>6$ Admissão $>14$ & 0,5594 & 0,4706 \\
\hline
\end{tabular}

Regressão Logística - Teste de Wald $\mathrm{p}<0,05$

Ao investigar a influência do resultado do parâmetro de avaliação de risco pré-operatório, não se rejeitou a hipótese nula de que a probabilidade de óbito com o parâmetro pré $\leq 6$ é igual a probabilidade de óbito com o parâmetro pré $>6(p=0,0622)$. Ao observar-se a hipótese nula de que a probabilidade de óbito com o parâmetro de admissão à UTI $\leq 14$ é igual à probabilidade de óbito com o parâmetro de admissão $>14$, o resultado do teste estatístico indicou a rejeição da hipótese nula no nível significativo de $5 \%(p=0,0012)$. A fim de analisar o resultado conjunto dos parâmetros de pré-operatório, peri-operatórios e de admissão à UTI, observou-se a hipótese nula de que a inclusão do parâmetro de admissão não tem influência na probabilidade de óbito na presença do parâmetro de admissão. Esta hipótese foi rejeitada no nível significativo de 5\% ( $p=0,0067$ ). Desta forma, a inclusão dos parâmetros de admissão à UTI melhora significativamente o prognóstico sobre a probabilidade de óbito do paciente.

Ao ajustar o modelo utilizando os dois parâmetros foi possível estimar as probabilidades de óbito do paciente, de acordo com os registros de parâmetros de risco nos dois momentos de avaliação. Na tabela 7 estão apresentadas as probabilidades estimadas pelo modelo e as proporções observadas no estudo.

Tabela 7 - Probabilidade de Óbito Estimada e Proporção de Óbito Observada.

\begin{tabular}{lcc}
\hline Parâmetros & $\begin{array}{c}\text { Probabilidade de } \\
\text { Óbito Estimada }\end{array}$ & $\begin{array}{c}\text { Proporção de } \\
\text { Óbito Observada }\end{array}$ \\
\hline Pré $\leq 6$ Admissão $\leq 14$ & 0,0356 & 0,0380 \\
Pré $\leq 6$ Admissão $>14$ & 0,1250 & 0,1111 \\
Pré $>6$ Admissão $\leq 14$ & 0,0576 & 0 \\
Pré $>6$ Admissão $>14$ & 0,1912 & 0,2353 \\
\hline
\end{tabular}

Regressão Logística - Teste de Wald $\mathrm{p}<0,05$

Uma análise desses resultados em conjunto com os valores de $p$ correspondentes aos testes estatísticos realizados permite concluir que o modelo estima ade- 
quadamente as probabilidades de óbito do paciente quando conhecidos os valores de seus parâmetros de pré-operatório, peri-operatórios e de admissão à UTI. Além disso, observou-se que quando o paciente apresentou um parâmetro na admissão à UTI mais favorável, ou seja, $\leq 14$, qualquer que tenha sido o seu parâmetro no pré-operatório, a sua probabilidade estimada de óbito é bem menor do que quando este apresenta um parâmetro de maior risco (>14) na admissão à UTI.

\section{DISCUSSÃO}

Segundo Daley ${ }^{23}$, o desenvolvimento de um modelo de risco para pacientes hospitalizados representa uma tarefa complexa cujos critérios a serem incluídos neste devem atender o objetivo a ser alcançado. Com enfoque no procedimento diagnóstico principal e a sua evolução, de modo a identificar aquele com maior risco de complicações, Hammermeister ${ }^{24}$ conferiu ao uso de modelos de risco e aos seus resultados como a uma contínua participação de desenvolvimento que objetive a coleta fiel de dados do prontuário.

O desenvolvimento de critérios para inclusão em programas de avaliação de resultados (risco do paciente) exige clara definição do tratamento períodos de pré, peri e pós-operatórios. Devem-se definir os resultados de interesse, o tempo de avaliação, as variáveis a serem analisadas, os recursos disponíveis para a coleta dos dados, o pesquisador que procede à coleta dos dados, a padronização dos dados coletados e como coletar no banco de dados, o período de análise, o número necessário de casos e a técnica de análise estatística. Após, observa a validação do método. Deve-se também saber como tratar os dados perdidos durante a coleta de informações, como bem destacou Daley ${ }^{23}$.

\section{Pré-Operatório}

Os fatores de risco são aqueles característicos do paciente que podem influenciar na mortalidade operatória. Estes fatores são necessariamente restritos aos resultados pré-operatórios e não podem incluir parâmetros peri e pós-operatórios ${ }^{25}$. Baseando-se na definição descrita, a partir de 1980 teve início o desenvolvimento de escalas de risco que visavam sobretudo a avaliação da mortalidade operatória ${ }^{10,26-30}$.

Dentre os parâmetros mais comuns para análise do pré-operatório, encontram-se a idade ${ }^{31-36}$, o sexo $0^{11,37-40}$, a função do $\mathrm{VE}^{41-44}$, as doenças co-mórbidas ${ }^{45,46}$, a angiografia coronária com aterosclerose proximal ou dis- tal ${ }^{47,48}$. Foram incluídas também cirurgias de urgência (relacionada intimamente com a angiografia coronária e a função do $\mathrm{VE}^{49}$ ) e doenças cirúrgicas valvares que comprometem a função ventricular esquerda (EAO, $\mathrm{IMt})^{12,50}$. A menor área de superfície corporal apresenta maior risco para síndrome de baixo débito cardíaco e mortalidade operatória, segundo Fisher e col. ${ }^{51}$, e também com Christakis e col. ${ }^{52}$.

$\mathrm{Na}$ análise dos resultados dos 400 pacientes estudados, a avaliação da escala de risco em pacientes com parâmetro abaixo ou igual a 6 e acima de 6 , não há diferença estatística no nível de significância de 5\% (p = 0,0622). Ou seja, os dados de pré-operatório são válidos para análise do modelo proposto.

\section{Admissão na UTI}

O uso de parâmetros para avaliação da morbidade e mortalidade à admissão na UTI iniciou-se com Knaus e col. através do protocolo APACHE ${ }^{53}$. A partir do desenvolvimento do APACHE II, Knaus e col. ${ }^{54}$, houve diminuição das variáveis fisiológicas de 34 para 12. Isso foi o mínimo avaliado para analisar alterações fisiológicas de todos os sistemas orgânicos mas que mantiveram precisão estatística.

Higgins e col. em $1997^{12}$, desenvolveram um método com a interação de variáveis de pré-operatório e perioperatório e à admissão na UTI. Essa análise incluiu dados de peri e pós-operatório imediato porque esses também podem influenciar a evolução do paciente com as técnicas cirúrgicas utilizadas ${ }^{55-59}$, o manuseio anestésico ${ }^{60-65}$, a revascularização completa ou não do miocárdio ${ }^{66,67}$, a proteção miocárdica ${ }^{68}$, e o manuseio hemodinâmico ${ }^{69-71}$.

Nos critérios de admissão à UTI incluíram-se dados do pré e peri-operatórios e o quadro fisiológico de admissão à UTI. O pré-operatório incluiu a idade acima de 70 anos, área de superfície corporal, doenças co-mórbidas e re-operação coronariana, angioplastia coronariana ou vascular periférica, alterações dos níveis de creatinina e de albumina e/ou hematócrito (anemia).

Os eventos de peri-operatório foram o tempo de CEC e o uso do balão intra-aórtico após a revascularização do miocárdio. O maior tempo de CEC proporcionou maior risco para complicações ${ }^{63,72,73}$ e o uso do balão intra-aórtico indiretamente relacionado com pior função miocárdica ${ }^{74-76}$. A inclusão de dados fisiológicos na escala de riscos na UTI esteve presente em dois protocolos: no APACHE e no desenvolvido na Cleveland Clinic Foundation (CCF), ambos com a participação de Knaus. Nos parâmetros fisiológicos, realizou-se a 
substituição do índice cardíaco pelo desempenho cardiovascular, este com utilização de critérios clínicos ${ }^{77}$. Estes dados do desempenho cardiovascular foram desenvolvidos anteriormente ${ }^{78-83}$.

$\mathrm{Na}$ avaliação dos casos de óbitos a presença dos dados de peri-operatório ou alterações no quadro fisiológico à admissão à UTI indicaram que os resultados foram superiores em precisão somente em relação à avaliação de pré-operatória ${ }^{12,53,54,84}$.

Para a operação de revascularização do miocárdio, vários trabalhos vêm demonstrando mudanças na população de pacientes, no melhor atendimento préoperatório e anestésico, assim como melhores resultados no pós-operatório ${ }^{85-90}$, apesar do aumento do risco clínico na admissão hospitalar ${ }^{91}$. Observou-se também melhora no armazenamento de dados ${ }^{92}$. No período de avaliação do ano 1999 a 2002, ocorreram mudanças importantes na cirurgia de revascularização do miocárdio no serviço de cirurgia cardíaca do HUC: a CEC sem pinçamento aórtico total, o uso do AAS no préoperatório e o aumento do número de operações sem CEC. Os resultados em longo prazo dessas mudanças ainda estão por vir.

Os resultados operatórios podem ser diferentes por vários fatores além do volume cirúrgico, como o número entre operações eletivas ou de emergência, recursos disponíveis, entre outros. Somente a aferição do resultado baseado no número de operações realizadas pode trazer uma incorreta avaliação entre diferentes instituições ${ }^{93,94}$.

A partir de 1988, Hlatky e col. ${ }^{95}$ iniciaram estudos para comparação entre as escalas de risco. Esse estudo comparou uma avaliação local com estudos mais amplos.

No estudo de Orr e col. ${ }^{96}$, foram observados dados de pacientes em diferentes escalas (quatro). Em relação à mortalidade, as escalas da CCF e New York ${ }^{28}$ subestimaram este fator, e que a New York State's New Cardiac Surgery Reporting System (NE) e Parsonnet superestimaram a mortalidade. Salientaram pontos importantes como erros (problema na determinação de variáveis, coleta de dados), cuidados com a estimativa de risco (sub ou superestimação), e os modelos empregados (variação de mortalidade, fatores operatórios). Estudando quatro modelos (STS) Ontário Province Risk Score (PACCN), French Score (PARIS), United Kingdom Society of Cardiothorac Surgeons algorithm (UK), Bridgewater e col. ${ }^{97}$ concluíram que os melhores resultados eram o de Parsonnet (apesar da superestimativa de mortalidade) e o da UK. Procuraram também demonstrar a importância da epidemiologia e do modo diferente de avaliação dos pacientes em diversos locais de atendimento.

Martinez-Alario e col. ${ }^{98}$ relataram que as escalas gerais de avaliação de gravidade empregadas em UTI (APACHE, Simplified Acute Physiology Score (SAPS) e Mortality Probability Models (MPM)), expressaram melhores resultados em pacientes cirúrgicos. Nashef e col. ${ }^{99}$ compararam a escala de risco EuroSCORE em seis países, demonstrando a importância da epidemiologia e das diferentes condutas de tratamento em pacientes nesses países. Geissler e col. ${ }^{100}$, utilizando seis escores, concluíram que para a avaliação de mortalidade, o EuroSCORE era mais preditivo e que para morbidade o protocolo da CCF era mais conclusivo.

Em 2002, Pinna-Pintor e col. ${ }^{101}$ concluíram que os modelos testados (Parsonnet, CCF, PARIS, EuroSCORE) eram ineficazes para predizer mortalidade em um determinado paciente.

A escolha do modelo de escala de risco utilizado em pacientes submetidos à cirurgia de revascularização do miocárdio, para o desenvolvimento do trabalho, baseou-se na maior precisão de resultados com a inclusão de parâmetros de pré e peri-operatórios e de admissão à UTI. Apesar de vários modelos de escala desenvolvidos, seja de uso local ou restrito a um único serviço de cirurgia cardíaca ${ }^{8,27,102-105}$, regionais ${ }^{29,106-110}$, nacionais ${ }^{10,26,111-113}$ e também internacionais ${ }^{30}$ utilizarem parâmetros pré-operatórios para a avaliação de risco operatório na operação de revascularização do miocárdio, somente a partir de $1997^{12}$ foi empregado dados de admissão à UTI (parâmetros de peri e pósoperatórios imediatos). Este modelo de escala de risco, quando foi utilizado no serviço de cirurgia cardíaca do HUC, demonstrou que há maior precisão na avaliação do paciente quando os dados de admissão à UTI foram incluídos.

Em conclusão, o acréscimo de dados de peri e de pósoperatório conferiram maior acuidade para a avaliação dos pacientes submetidos à cirurgia de revascularização do miocárdio.

Anexo 1 - Cálculo de Fração de Ejeção

\footnotetext{
$\mathrm{FE}=\mathrm{VdVE}-\mathrm{VsVE} / \mathrm{VdVE}$

$\mathrm{FE}=$ fração de ejeção

VdVE = volume diastólico do ventrículo esquerdo

VsVE = volume sistólico do ventrículo esquerdo

FE normal maior que 0,65

FE com diminuição discreta $0,55-0,65$

FE com diminuição moderada $0,45-0,55$

FE com diminuição importante menor que 0,45
} 
Anexo 2 - Cálculo do Encurtamento Percentual

$\mathrm{EP}=\mathrm{DdVE}-\mathrm{DsVE} / \mathrm{DdVE}$

$\mathrm{EP}=$ encurtamento percentual

DdVE = diâmetro diastólico do ventrículo esquerdo

DsVE = diâmetro sistólico do ventrículo esquerdo

EP normal maior que $30 \%$

EP com diminuição discreta $25 \%-30 \%$

EP com diminuição moderada $20 \%-25 \%$

EP com diminuição importante menor que $20 \%$

Anexo 3 - Cálculo da Diferença Alvéolo-Arterial de Oxigênio.

$\mathrm{P}(\mathrm{A}-\mathrm{a}) \mathrm{O}_{2}=\left(\mathrm{Pb}-\mathrm{PH}_{2} \mathrm{O}\right) \times \mathrm{FiO}_{2}-\left(\mathrm{PaO}_{2}+\mathrm{PaCO}_{2}\right)$

$\mathrm{Pb}=$ pressão barométrica

$\mathrm{PH}_{2} \mathrm{O}=$ pressão vapor de água no alvéolo $( \pm 47 \mathrm{mmHg})$

$\mathrm{FiO}_{2}$ = fração de oxigênio inspirado $(0,21 \mathrm{em}$ ar ambiente)

$\mathrm{PaO}_{2}=$ pressão alveolar de oxigênio

$\mathrm{PaCO}_{2}=$ pressão alveolar de gás carbônico

Anexo 4 - Quantificação dos Critérios de Identificação do Desempenho Cardiovascular ${ }^{77}$

1) Temperatura das extremidades:

- Quente

- Tépida ou morna

- Fria

2) Diferença entre a temperatura do joelho:

- Joelho mais frio do que o pé

- Joelho na mesma temperatura do pé

- Joelho mais quente do que o pé

3) Cor das extremidades:

- Corada

- Pálida

- Moteada

- Cianótica

4) Pulsos pediosos (tibial anterior):

- $4++++$

- $3+++$

- $2++$

- $1+$

- Ausentes

5) Pressão arterial:

- Normal

- mais de $25 \%$ acima do normal

- mais de $10 \%$ abaixo do normal

6) Fluxo urinário

- Maior do que $1 \mathrm{~mL} / \mathrm{kg} / \mathrm{h}$

- Menor do que $1 \mathrm{~mL} / \mathrm{kg} / \mathrm{h}$

7) Sudorese

Intensa (mais do que uma área corpórea)

- Moderada

- Discreta

- Ausente

8) Ansiedade

- Agitação

- Confusão mental

- Distúrbio psiconeurótico

- Ausente

9) Instabilidade elétrica-ventricular

- Presença de extrassístole ventricular multifocal

- Presença de períodos de taquicardia ventricular

- Ausente
Anexo 5 - Graduação do Pulso Pedioso ${ }^{77}$

Pulso + pulso débil, não bem definido à palpação

Pulso ++ pulso bem definido à palpação, mas ainda débil

Pulso +++ pulso cheio, bem definido à palpação

Pulso ++++ pulso cheio, bem definido à palpação, empurra o dedo que palpa, em cada sístole

Anexo 6 - Categorias do Desempenho Cardiovascular - Critérios Clínicos $^{77}$

Desempenho Critérios Clínicos

Cardiovascular Pulsos Pés T. PA FU

Cor T. Joelho x Pés $(\mathrm{mL} / \mathrm{kg} / \mathrm{h})$

Adequado

- +++ ou corados quentes

- pés normais, mas maior que 1

- ++++ quentes $25 \%$ acima

Marginal

- ++ pálido, frios, iguais, normal menor que 1

- Inadequado, mais ou menos pálidos e frios; pés mais abaixo de ausente

- ausentes moteados e frios $10 \%$ do normal

$\mathrm{T}=$ temperatura; $\mathrm{PA}=$ pressão arterial; $\mathrm{FU}$ = fluxo urinário.

Anexo 7 - Correlação entre Categorias de Desempenho Cardiovascular e Medidas Hemodinâmicas Diretas.

\begin{tabular}{|c|c|}
\hline Desempenho Cardiovascular & $\begin{array}{l}\text { Medida Hemodinâmica Direta } \\
\text { Índice cardíaco }(\mathrm{L} / \mathrm{min} / \mathrm{m} 2)\end{array}$ \\
\hline Adequado & maior ou igual a $2 \mathrm{~L} / \mathrm{min} / \mathrm{m} 2$ \\
\hline Marginal & $\begin{array}{l}\text { maior ou igual a } 1,6 \text { e menor } \\
\text { que } 2 \mathrm{~L} / \mathrm{min} / \mathrm{m} 2\end{array}$ \\
\hline Inadequado & menor que $1,6 \mathrm{~L} / \mathrm{min} / \mathrm{m} 2$ \\
\hline
\end{tabular}

\section{REFERÊNCIAS}

01. Favaloro RG - Saphenous vein autograft replacement of severe segmental coronary artery occlusion - Operative technique. Ann Thorac Surg, 1968; 05:334-339.

02. Favaloro RG -Saphenous vein graft in the surgical treatment of coronary artery disease - Operative technique. J Thorac Cardiovasc Surg, 1969;58:178-185.

03. Ankeney $\mathrm{JL}$ - To use or not to use the pump oxygenator in coronary bypass operations. Ann Thorac Surg, 1975;19:108-109.

04. Buffolo E, Andrade JCS, Succi JE et al - Revascularização direta do miocárdio sem circulação extracorpórea. Descrição da técnica e resultados iniciais. Arq Bras Cardiol, 1982;38:365-373.

05. Ferguson Jr,TB, Hammill BG, Peterson ED, et al. A decade of change-risk profiles and outcomes for isolated coronary artery bypass grafting procedures, 1990-1999: a report from the STS National Database Committee and the Duke Clinical Research Institute. Ann Thorac Surg, 2002;73:480-490.

06. Kirklin JW - Guidelines and indications for coronary artery bypass graft surgery. A report of the American College of Cardiology/American Heart Association Task Force on assessment of diagnostic and therapeutic cardiovascular procedures (Subcommittee on coronary artery bypass graft surgery ). J Am Coll Cardiol, 1991;17:543-589.

07. Eagle KA, Guyton RA, Davidoff R et al - ACC/AHA Guidelines for coronary bypass graft surgery: executive summary and recommendations. A report of the American College of Cardiology/American Heart Association task force on practice guidelines (Committee to revise the 1991 guidelines for coronary artery bypass graft surgery). Circulation, 1999;100:1464-1480.

08. Higgins TL, Estafanous FG, Loop FD et al - Stratification of morbidity and mortality outcome by preoperative risk factors in coronary artery bypass patients. A clinical severity score. JAMA, 1992;267:2344-2348.

09. Kollef MH, Schuster DP - Previsão do prognóstico na UTI com sistemas de 
graduação. Conceitos e princípios básicos, em: Carlson RW, Geheb MA. Clínicas de terapia intensiva. Rio de Janeiro: Interlivros 1994;1:1-19.

10. Edwards FH, Clark RE, Schwartz M - Coronary artery bypass grafting: The Society of Thorac Surgeons national database experience. Ann Thorac Surg, 1994;57:12-19.

11. Daly LE, Lonergan NM, Graham I-Predicting operative mortality after coronary artery bypass surgery in males. Quaterly J Medicine, 1993;86:771-778.

12. Higgins TL, Estafanous FG, Loop FD et al - ICU admission score for predicting morbidity and mortality risk after coronary artery bypass grafting. Ann Thorac Surg, 1997;64:1050-1058.

13. Aloan L - Apêndice 3 - Superfície corporal. In: Aloan L. Hemodinâmica e angiocardiografia - Obtenção de dados, interpretação, aplicações clínicas. $2^{\mathrm{a}}$ Ed, Rio de Janeiro: Atheneu, 1990; 621-622.

14. Pombo JF, Troy BL, Russell Jr. RO - Left ventricular volumes and ejection fraction by echocardiography. Circulation, 1971; 43: 480-490.

15. Teichholz LE, Kreulen T, Herman MV, Gorlin R - Problems in echocardiographic volume determinations: echocardiographic-angiographic correlations in the presence or absence of asynergy. Am J Cardiol, 1976;37:7-11.

16. Feigenbaum $\mathrm{H}$ - Avaliação Ecocardiográfica das Câmaras Cardíacas, em: Feigenbaum H - Ecocardiografia. 4a Ed, São Paulo: Roca, 1988; 141-245.

17. Aloan $L$ - Função ventricular, em: Aloan $L$-Hemodinâmica e Angiocardiografia - Obtenção de Dados, Interpretação, Aplicações Clínicas. $2^{a}$ Ed, Rio de Janeiro: Atheneu, 1990;116-163.

18. Braunwald DE - Assessment of Cardiac Surgery, em: Braunwald DE - Heart Disease. A Textbook of Cardiovascular Medicine. Philadelphia: W.B. Saunders Company, 1992;419-443.

19. Fann JI, Ingels Jr WB, Muller DC - Pathophysiology of Mitral Valve Disease and Operative Indications, em: Edmunds Jr LM - Cardiac surgery in the adult. New York, McGraw-Hill, 1997, 959-990.

20. Maier GW \& Wechsler AS - Pathophysiology of Aortic Valve Disease, em: Edmunds Jr LH- Cardiac surgery in the adult. New York, McGraw-Hill, 1997; 835-858.

21. McCarthy PM, Golding LAR - Temporary mechanical circulatory support, em: Edmunds Jr LH - Cardiac surgery in the adult. New York, McGraw-Hill, 1997;319-338.

22. Gonçalves Jr I, Knobel E, Lorenzi GF et al - Fórmulas, Tabelas e Valores de Referência Utilizados em Terapia Intensiva, em - Knobel E - Condutas no Paciente Grave. São Paulo, Atheneu, 1997; 856-883.

23. Daley $\mathrm{J}$ - Criteria by which to evaluate risk-adjusted outcomes programs in cardiac surgery. Ann Thorac Surg 1994, 58: 1827-1835.

24. Hammermeister KE - Participatory continuous improvement. Ann Thorac Surg, 1994;58:1815-1821.

25. Edwards FH, Grover FL - Surgical Risk Assessment, em: Karp RB, Laks H, Wechsler AS - Advances in Cardiac Surgery. St.Louis: Mosby; 2000;77-96.

26. Kennedy JW, Kaiser GC, Fisher LD et al - Multivariate discriminant analysis of the clinical and angiographic predictors of operative mortality from the Collaborative Study in Coronary Artery Surgery (CASS). J Thorac Cardiovasc Surg, 1980;80:876-887.

27. Parsonnet $V$, Dean D, Bernstein AD - A method of uniform stratification of risk for evaluating the results of surgery in acquired adult heart disease. Circulation, 1989;79:(Suppll):I3-112.

28. Hannan EL, Kilburn Jr H, O'Donnell JF et al - Adult open heart surgery in New York State. An analysis of risk factors and hospital mortality rates. JAMA, 1990;264:2768-2774.

29. O'Connor GT, Plume SK, Olmstead EM et al - A regional prospective study of in-hospital mortality associated with coronary artery bypass grafting. JAMA, 1991;266:803-809.

30. Nashef SA, Roques F, Michel P et al - European system for cardiac operative risk evaluation (EuroSCORE). Eur J Cardiothorac Surg, 1999;16:9-13.

31. Weintraub WS, Craver JM, Cohen CL et al - Influence of age on results of coronary artery surgery. Circulation, 1991;84:(Suppll5):III226-III235.

32. Khan SS, Kupfer JM, Matloff JM et al - Interaction of age and preoperative risk factors in predicting operative mortality for coronary bypass surgery. Circulation, 1992;86:(Suppll15):II186-II190.

33. Ko W, Gold JP, Lazzaro R et al - Survival analysis of octogenarian with coronary artery disease managed by elective coronary artery bypass surgery versus conventional medical treatment. Circulation, 1992;86:(Suppl5):I1191-II197.

34. Silva LHF, Nascimento CS, Viotti LAP Jr - Revascularização do miocárdio em idosos. Rev Bras Cir Cardiovasc, 1997;12:132-140.

35. Iglezias JCR, Oliveira Jr JL, Fels KW et al - Fatores prognósticos na revascularização do miocárdio em pacientes idosos. Rev Bras Cir Cardiovasc, 1997;12:325-334.
36. Yamamuro M, Lytle BW, Sapp SK et al - Risk factors and outcomes after coronary reoperation in 739 elderly patients. Ann Thorac Surg, 2000;69:464-474.

37. Carey JS, Cukingnan RA, Singer LK - Health status after myocardial revascularization: inferior results in women. Ann Thorac Surg, 1995;59:112-117.

38. Aldea GS, Gaudiani JM, Shapira OM et al - Effect of gender on postoperative outcomes and hospital stays after coronary artery bypass grafting. Ann Thorac Surg, 1999;67:1097-1103.

39. Abramov D, Tamariz MG, Sever JY et al - The influence of gender on the outcome of coronary artery bypass surgery. Ann Thorac Surg, 2000;70:800-806.

40. O'Rourke DJ, Malenka DJ, Olmstead EM et al - Improved in-hospital mortality in women undergoing coronary artery bypass grafting. Ann Thorac Surg, 2001;71:507-511.

41. Young Jr WG, Sabiston Jr DC, Ebert PA et al - Preoperative assessment of left ventricular function in patients selected for direct myocardial revascularization. Ann Thorac Surg, 1971;11: 395-402.

42. Jones EL, Craver JM, Kaplan JA et al - Criteria for operability and reduction of surgical mortality in patients with severe left ventricular ischemia and dysfunction. Ann Thorac Surg, 1978;25:413-424.

43. Goenen M, Jacquemart JL, Galvez $\mathrm{S}$ et al - Preoperative left ventricular dysfunction and operative risks in coronary bypass surgery. Chest, 1987;92:804-806.

44. Oliveira SF, Jatene $A D$, Solimene $M C$ et al - Cirurgia de revascularização do miocárdio em pacientes com cardiomiopatia isquêmica e disfunção ventricular acentuada: resultados imediatos e de longo prazo. Rev Bras Cir Cardiovasc, 1997;12:1-9.

45. Charlson ME, Pompei P, Ales KL et al - A new method of classifying prognostic comorbidity in longitudinal studies: development and validation. $J$ Chronic Dis, 1987;40:373-383.

46. Jencks SF, Williams DK, Kay TL - Assessing hospital-associated deaths from discharge data. The role of length of stay and comorbities. JAMA, 1988;260:2240-2246

47. Friesinger GC, Page EE, Ross RS - Prognostic significance of coronary arteriography. Trans Assoc Am Physicians, 1970;83:78-92.

48. Graham MM, Chambers RJ, Davies RF - Angiographic quantification of diffuse coronary artery disease: reliability and prognostic value for bypass operations. J Thorac Cardiovasc Surg, 1999;118:618-627.

49. Teoh KH, Christakis GT, Weisel RD et al - Increased risk of urgent revascularization. J Thorac Cardiovasc Surg, 1987;93:291-299.

50. Lytle BW, Cosgrove DM, Loop FD et al - Replacement of aortic valve combined with myocardial revascularization: determinants of early and late risk for 500 patients, 1967-1981. Circulation, 1983;68:1149-1162.

51. Fisher LD, Kennedy JW, Davis KB et al - Association of sex, physical size, and operative mortality after coronary artery bypass in the Coronary Artery Surgery Study (CASS). J Thorac Cardiovasc Surg, 1982;84:334-341.

52. Christakis GT, Weisel RD, Buth KJ et al - Is body size the cause for poor outcomes of coronary artery bypass operations in women? J Thorac Cardiovasc Surg, 1995;110:1344-1358.

53. Knaus WA, Zimmerman JE, Wagner DP et al - APACHE - acute physiology and chronic health evaluation: a physiologically based classification system. Crit Care Med, 1981;9:591-597.

54. Knaus WA, Draper EA, Wagner DP et al - APACHE II: A severity of disease classification system. Crit Care Med, 1985;13:818-829.

55. Metcalfe MJ, Lip GY, Dargie HJ - Factors influencing coronary artery bypass graft patency. Cardiovascular Surgery, 1994;2:679-685.

56. Wechsler AS - Implication of myocardial lactate metabolism during CABG (Ando et al.). Cardiovasc Surgery, 1997;5:208-209.

57. Ando $\mathrm{H}$, Tanaka J, Hisahara $\mathrm{M}$ et al - Implication of myocardial lactate metabolism during coronary artery bypass grafting. Cardiovasc Surgery, 1997;5:210-215.

58. Baumgartner FJ, Gheissari A, Capouya ER et al - Technical aspects of total revascularization in off-pump coronary bypass via sternotomy approach. Ann Thorac Surg, 1999;67:1653-1658.

59. Cleveland Jr JC, Shroyer AL, Chen AY et al - Off-pump coronary artery bypass grafting decreases risk-adjusted mortality and morbidity. Ann Thorac Surg, 2001;72:1282-1289.

60. Charlson ME, MacKenzie CR, Gold JP et al - The preoperative and intraoperative hemodynamic predictors of postoperative myocardial infarction or ischemia in patients undergoing noncardiac surgery. Ann Surg, 1989;210:637-648.

61. Merry AF, Ramage MC, Whitlock RM et al - First-time coronary artery bypass grafting: the anaesthetist as a risk factor. Br J Anaesth, 1992;68:6-12. 
62. Baur HR, Peterson TA, Arnar O et al - Predictors of perioperative myocardial infarction in coronary artery operation. Ann Thorac Surg, 1981;31:36-44.

63. Edmunds LH Jr. - Why Cardiopulmonary Bypass Makes Patient Sick: Strategies to Control the Blood-Synthetic Surface Interface, em: Karp RB, Laks H, Wechsler AS - Advances in Cardiac Surgery. St. Louis, Mosby, 1995;131-167.

64. Boyle EM Jr, Pohlman TH, Johnson MC et al. Endothelial cell injury in cardiovascular surgery: the systemic inflammatory response. Ann Thorac Surg, 1997;63:277-284.

65. Menasché $\mathrm{P}$ - The systemic factor: the comparative roles of cardiopulmonary bypass and off-pump surgery in the genesis of patient injury during and following cardiac surgery. Ann Thorac Surg, 2001;72:S2260-S2266.

66. Jones EL, Weintraub WS - The importance of completeness of revascularization during long-term follow-up after coronary artery operations. J Thorac Cardiovasc Surg, 1996;112:227-237.

67. Scott R, Blackstone EH, McCarthy PM et al - Isolated bypass grafting of the left internal thoracic artery to the left anterior descending coronary artery: late consequences of incomplete revascularization. J Thorac Cardiovasc Surg, 2000;120:173-184.

68. Loop FD, Higgins TL, Panda R et al - Myocardial protection during cardiac operations. Decreased morbidity and lower cost with blood cardioplegia and coronary sinus perfusion. J Thorac Cardiovasc Surg, 1992;104:608-618.

69. Slogoff S, Keats AS - Does perioperative myocardial ischemia lead to postoperative myocardial infarction? Anesthesiology, 1985;62:107-114.

70. Reich DL, Bodian CA, Krol M et al - Intraoperative hemodynamic predictors of mortality, stroke, and myocardial infarction after coronary artery bypass surgery. Anesth Analg, 1999;89: 814-822.

71. Lima RC, Escobar MAS, Della Santa RF et al - Avaliação hemodinâmica intra-operatória na cirurgia de revascularização miocárdica sem auxílio da circulação extracorpórea. Rev Bras Cir Cardiovasc, 2000;15:210-211.

72. Butler J, Rocker GM, Westaby S - Inflammatory response to cardiopulmonary bypass. Ann Thorac Surg, 1993;55:552-559.

73. Taylor KM - SIRS - The systemic inflammatory response syndrome after cardiac operations. Ann Thorac Surg, 1996;61:1607-1608.

74. Bavaria JE, Furukawa S, Kreiner $\mathrm{G}$ et al - Effect of circulatory assist devices on stunned myocardium. Ann Thorac Surg, 1990;49:123-128.

75. Pi K, Block PC, Warner MG et al - Major determinants of survival and nonsurvival of intraaortic balloon pumping. Am Heart J, 1995;130: 849-853.

76. Kang N, Edwards $\mathrm{M}$, Larbalestier R - Preoperative intraaortic balloon pumps in high-risk patients undergoing open heart surgery. Ann Thorac Surg, 2001;72:54-57.

77. Lucchese FA - Identificação do Desempenho Cardiovascular, em: Lucchese FA - Tratamento Intensivo Pós-Operatório. São Paulo, Fundo Editorial BYK, 1987;45-53.

78. Joly HR, Weil MH - Temperature of the great toe as an indication of the severity of shock. Circulation, 1969;39:131-138.

79. Henning RJ, Wiener F, Valdes $\mathrm{S}$ et al - Measurement of toe temperature for assessing the severity of acute circulatory failure. Surg Gynecol Obstet, 1979;149:1-7.

80. Kirklin JK, Kirklin JW - Management of the cardiovascular subsystem after cardiac surgery. Ann Thorac Surg, 1981;32:311-319.

81. Moraes LB, Holzhaker RA, Lucchese FA - Técnica para determinação indireta do desempenho cardiovascular através da medida da temperatura de extremidades em pós-operatório de cirurgia cardíaca. Arq Bras Cardiol, 1981;37:50.

82. Kirklin JK, Blackstone EH, Kirklin JW et al - Intracardiac surgery in infants under age 3 months: predictors of postoperative in-hospital cardiac death Am J Cardiol, 1981; 48:507-512.

83. Bailey JM, Levy JH, Kopel MA et al - Relationship between clinical evaluation of peripheral perfusion and global hemodynamics in adults after cardiac surgery. Crit Care Med, 1990;18:1353-1356.

84. Knaus WA, Wagner DP, Draper EA et al - The APACHE III prognostic system. Risk prediction of hospital mortality for critically ill hospitalized adults. Chest,1991:100:1619-1636.

85. Rahimtoola SH, Grunkemeier GL, Teply JF et al - Changes in coronary bypass surgery leading to improved survival. JAMA, 1981;246:1912-1916.

86. Naunheim KS, Fiore AC, Wadley JJ et al - The changing profile of the patient undergoing coronary artery bypass surgery. J Am Coll Cardiol, 1988:11:494-498.

87. Califf RM, Harrell FE Jr, Lee KL et al - The evolution of medical and surgical therapy for coronary artery disease. A 15 year perspective. JAMA, 1989;261: 2077-2086.

88. Christakis GT, Ivanov J, Weisel RD et al - The changing pattern of coronary artery bypass surgery. Circulation, 1989;80:I-151-I161.
89. Weintraub WS, Jones EL, King SB $3^{\text {rd }}$ et al - Changing use of coronary angioplasty and coronary bypass surgery in the treatment of chronic coronary artery disease. Am J Cardiol, 1990;65:183-188.

90. Warner CD, Weintraub WS, Craver JM et al - Effect of cardiac surgery patient characteristics on patient outcomes from 1981 through 1995. Circulation, 1997;96:1575-1579.

91. Estafanous FG, Loop FD, Higgins TL et al - Increased risk and decreased morbidity of coronary artery bypass grafting between 1986 and 1994. Ann Thorac Surg, 1998;65:383-389.

92. Ferguson TB Jr, Dziuban Jr SW, Edwards FH et al - The STS National Database: current changes and challenges for the new millennium. Ann Thorac Surg, 2000;69:680-691.

93. Luft HS, Bunker JP, Enthoven AC - Should operations be regionalized ? The empirical relation between surgical volume and mortality. $\mathrm{N}$ Engl J Med, 1979;301:1364-1369.

94. Flood AB, Scott WR, Ewy W - Does practice make perfect? Part I: the relation between hospital volume and outcomes for selected diagnostic categories. Med Care, 1984;22:98-114.

95. Hlatky MA, Califf RM, Harrell FE Jr et al - Comparison of predictions based on observational data with the results of randomized controlled clinical trials of coronary artery bypass surgery. J Am Coll Cardiol, 1988;11:237-245.

96. Orr RK, Maini BS, Sottile FD et al - A comparison of four severity-adjusted models to predict mortality after coronary artery bypass graft surgery. Arch Surg, 1995;130:301-306.

97. Bridgewater $\mathrm{B}, \mathrm{Neve} \mathrm{H}, \mathrm{Moat} \mathrm{N}$ et al - Predicting operative risk for coronary artery surgery in the United Kingdom: a comparison of various risk prediction algorithms. Heart, 1998;79:350-355.

98. Martinez-Alario J, Tuesta ID, Plasencia E et al - Mortality prediction in cardiac surgery patients. Comparative performance of Parsonnet and General Severity Systems. Circulation, 1999;99:2378-2382.

99. Nashef SA, Roques F, Michel P et al - Coronary surgery in Europe: comparison of the national subsets of the European System for Cardiac Operative Risk Evaluation database. Eur J Cardiothorac Surg, 2000;17:396-399.

100. 100.Geissler HJ, Hölzl P, Marohl S etal-Risk stratification in heartsurgery: comparison of six score systems. Eur J Cardiothorac Surg, 2000;17:400-406.

101. 101.Pinna-Pintor P, Bobbio M, Colangelo $S$ et al - Inaccuracy of four coronary surgery risk-adjusted models to predict mortality in individual patients. Eur J Cardiothorac Surg, 2002;21:199-204.

102. Loop FD, Berrettoni JN, Pichard A et al - Selection of the candidate for myocardial revascularization. A profile of high risk based on multivariate analysis. J Thorac Cardiothorac Surg, 1975;69:40-51.

103. Wright JG, Pifarré R, Sullivan $\mathrm{HJ}$ et al - Multivariate discriminant analysis of risk factors for operative mortality following isolated coronary artery bypass graft. Loyola University Medical Center experience, 1970 to 1984. Chest, 1987:91:394-399.

104. Iyer VS, Russel WJ, Leppard P et al - Mortality and myocardial infarction after coronary artery surgery. A review of 12003 patients. Med J Aust, 1993;159:166-170.

105. Weintraub WS, Wenger NK, Jones EL et al - Changing clinical characteristics of coronary surgery patients. Differences between men and women. Circulation, 1993:88:II79-1I86.

106. Williams SV, Nash DB, Goldfarb N - Differences in mortality from coronary artery bypass graft surgery at five teaching hospitals. JAMA, 1991;266:810-815.

107. Hannan EL, Kilburn H Jr, Racz M et al - Improving the outcomes of coronary artery bypass surgery in New York State. JAMA, 1994;271:761-766.

108. Tu JV, Jaglal SB, Naylor CD et al - Multicenter validation of a risk index for mortality, intensive care unit stay, and overall hospital length of stay after cardiac surgery. Circulation, 1995;91:677-684.

109. Arom KV, Petersen RJ, Orszulak TA et al - Establishing and using a local/ regional cardiac surgery dabase. Ann Thorac Surg, 1997;64:1245-1249.

110. Holman WL, Allman RM, Sansom M et al - Alabama coronary artery bypass grafting project. Results of a statewide quality improvement initiative. JAMA, 2001;285:3003-3010.

111. Grover FL, Johnson RR, Marshall G et al - Factors predictive of operative mortality among coronary artery bypass subsets. Ann Thorac Surg 1993:56:1296-1307.

112. Ghali WA, Quan H, Brant R - Coronary artery bypass grafting in Canada: national and provincial mortality trends, 1992-1995. CMAJ, 1998;159:25-31.

113. Mozes B, Olmer L, Galai N et al - A national study of postoperative mortality associated with coronary artery bypass grafting in Israel. Ann Thorac Surg, 1998;66:1254-1263. 